EPJ Web of Conferences 41, 05016 (2013)

DOI: $10.1051 /$ epjconf/20134105016

(C) Owned by the authors, published by EDP Sciences, 2013

\title{
Ultrafast ignition of a uni-directional molecular motor
}

Jamie Conyard, ${ }^{1}$ Kiri Addison, ${ }^{1}$ Ismael A. Heisler, ${ }^{1}$ Arjen Cnossen, ${ }^{2}$ Wesley R. Browne, ${ }^{2}$ Ben L.

Feringa $^{2}$ and Stephen R. Meech ${ }^{1}$

${ }^{a}$ School of Chemistry, University of East Anglia, Norwich Research Park, Norwich NR4 7TJ

${ }^{\mathrm{b}}$ Stratingh Institute for Chemistry, University of Groningen, Nijenborgh 4, 9747AG Groningen, The

Netherlands

\begin{abstract}
Light-driven molecular motors convert light into mechanical energy via excited state reactions. In this work we follow sub-picosecond primary events in the cycle of a two-stroke unidirectional motor by fluorescence up-conversion and transient absorption.
\end{abstract}

\section{Introduction}

Light driven molecular machines are designed to convert photon energy into mechanical energy, which can often be achieved through an excited state isomerisation reaction. For the operation of molecular motors it is also clearly essential to exercise control over the direction of motion. Unidirectional operation of light-driven molecular motors was first reported by Feringa and coworkers in their studies of chiral overcrowded alkenes (Fig. 1)[1]

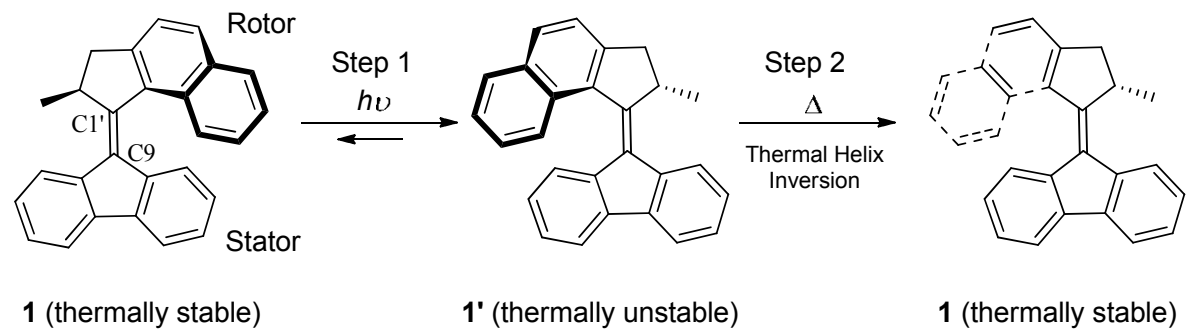

Figure 1 Structure of the molecular motor studied, indicating the rotor and stator of the motor linked by the double bond axle. The initial photochemical step is followed by the thermal stroke

In these molecules absorption gives rise to an isomerisation step which is followed by a thermal inversion in which the configuration at the stereogenic centre leads to rotation with an overwhelming preference for one direction. Subsequent sequential photo- and thermal steps result in one complete cycle. Key questions to be addressed include the rate of the isomerisation, its sensitivity to solvent friction, the extent to which the reaction occurs on the excited state surface and the possibility of controlling the rate of isomerisation through the use of shaped pulses.

This is an Open Access article distributed under the terms of the Creative Commons Attribution License 2.0, which permits unrestricted use, distribution, and reproduction in any medium, provided the original work is properly cited. 


\section{Results and Discussion}

Time resolved fluorescence was measured at wavelengths spanning the emission spectrum. The decay on blue edge is ultrafast, revealing components on the order of $100 \mathrm{fs}$. As the observation wavelength increases the decay time lengthens, but no risetime is registered on the red edge of the spectrum (Fig. 2). Superimposed on the fast fluorescence decay is an oscillatory component which can be analysed in terms of one highly damped ( $90 \mathrm{fs})$ low frequency mode $\left(113 \mathrm{~cm}^{-1}\right)$ and one less damped (220 fs) higher frequency mode $\left(180 \mathrm{~cm}^{-1}\right)$; these are illustrated in the Fourier transform (inset). Such oscillations in the fluorescence are characteristic of vibrational modes in the excited state which are displaced with respect to the ground state. Ultrafast fluorescence is particularly well suited to the detection of such excited state modes

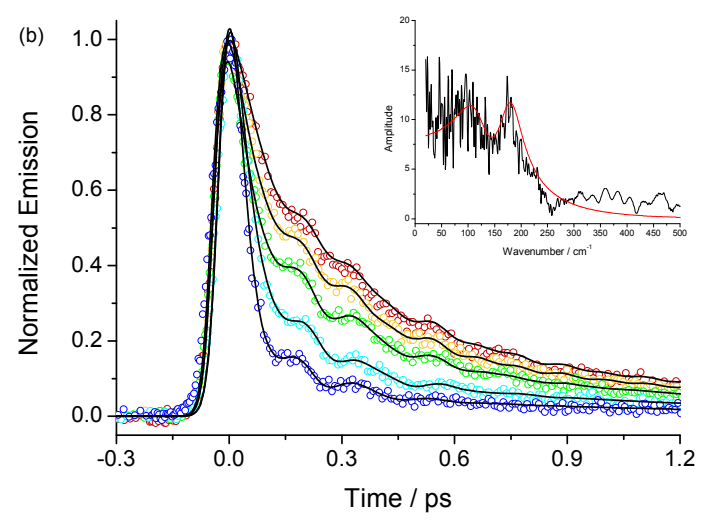

Figure. 2. Time resolved fluorescence observed for the motor molecule of Fig. 1. Wavelengths span the spectrum from $455 \mathrm{~nm}$ (dark blue) to $556 \mathrm{~nm}$ (red). The inset shows a Fourier transform of the oscillatory part of the fluorescence.

The wavelength resolved time resolved fluorescence can be converted into time dependent emission spectra, which reveal that the spectral mean frequency shifts to the red as the integrated emission intensity collapses on a time scale of a few hundred femtoseconds. Subsequent to these fast events the fluorescence decays with a time constant of $1.4 \mathrm{ps}$. The oscillatory features evident in the wavelength resolved data of Fig. 2 translate to an oscillation in both the intensity and the mean frequency of the time resolved spectrum[2]. This shows that the low frequency modes excited are coupled to the electronic transition moment.

Transient absorption measurements reveal two spectral features (Fig. 3) with distinct kinetics. The lowest energy one is formed rapidly (within the $300 \mathrm{fs}$ time resolution of the apparatus) and
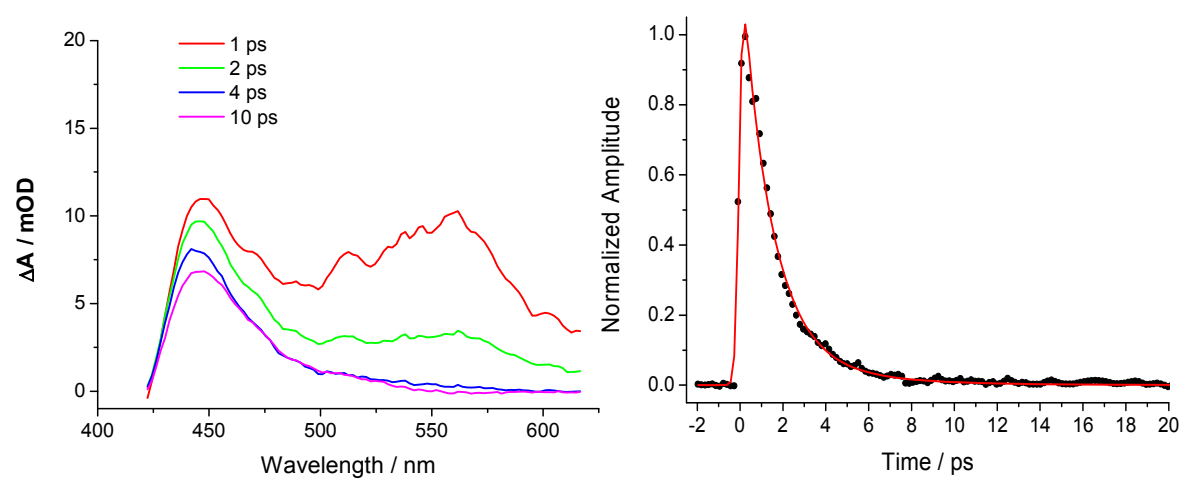

Figure 3. Transient absorption data for the motor in the visible region 
decays with a $1.5 \pm 0.3$ ps decay constant. The higher energy feature is evidently a mixture of the 1.5 ps transient, stimulated emission a very long lived (>100 ps) transient and a ground state bleach. We assign the 1.5 ps state to the relaxed excited state observed in the fluorescence data.

Based on these data we assign the primary events in the photoactivated stroke of a molecular motor following electronic excitation as follows. The bright Franck-Condon excited state relaxes in a few hundred femtoseconds to a less bright relaxed state. The high rate suggests a small scale energetically downhill structural transformation, possible involving pyramidalization at the C9 carbon, as suggested by quantum chemical calculation[3]. The relaxed state populated in ca $200 \mathrm{fs}$ and relaxes back to the ground state in $1.5 \mathrm{ps}$. Coherently excited vibrational modes modulate the transition moment, connecting the dark and bright $(\mathrm{F}-\mathrm{C})$ states. These assignments are illustrated schematically in Fig. 4. It is interesting that the coherently excited low frequency modes relax on the sub $100 \mathrm{fs}$ time scale, setting an upper limit to the time available for exerting coherent control over the primary photochemical step.

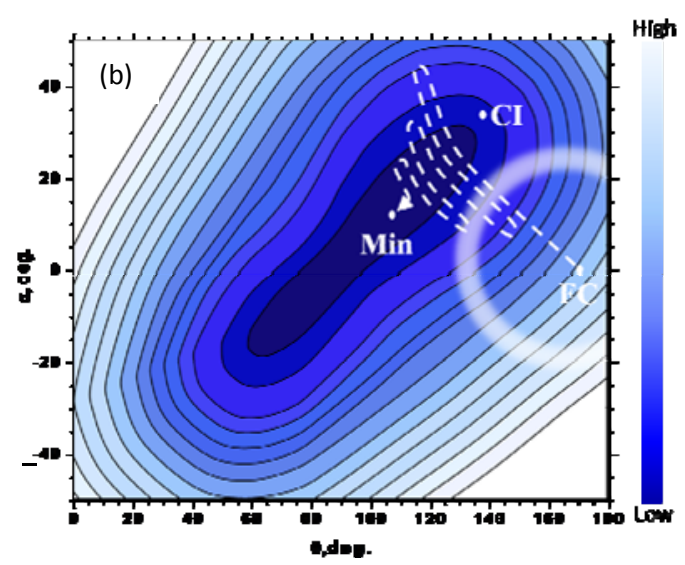

Figure 4. Schematic representation of the primary steps in a molecular motor

One route to the optical control of motor efficiency is through shaped light pulses, which may direct the excited state population towards or away from a conical intersection. However, the very fast damping of the lowest frequency modes suggests that the timescale in which this can be achieved is small. As an alternative to optical control of the rate and efficiency of motor operation we have investigated the effect of chemical modulation. Electron withdrawing (cyano, chloro) and donating (methoxy) groups were substituted on the 4' position on the naphthalenic ring. The effect of the substitution on the ground state structure was assessed. The data are complex but electron withdrawing groups accelerate the decay of the FC state, but extend the lifetime of the dark state. These data are being analysed in terms of modifications to the shape of the PES such as that described in Figure 4.

\section{References}

[1] N. Koumura, R. W. J. Zijlstra, R. A. van Delden, N. Harada, and B.L. Feringa Light-driven monodirectional molecular rotor. Nature 401 (6749), 152 (1999)

[2] J. Conyard, K. Addison, I. A. Heisler, A. Cnossen, W. R. Browne, B L. Feringa and S. R. Meech Ultrafast Dynamics in the Ignition Phase of Molecular Rotary Motors, Nature Chem. 4, 457 (2012).

[3] Kazaryan, A. et al., Understanding the Dynamics Behind the Photoisomerization of a LightDriven Fluorene Molecular Rotary Motor. J. Phys. Chem. A 114, 5058 (2010) 